\title{
A Broadband Millimeter-Wave Hollow Waveguide Coupling Structure Configured by a Wire-Eroded Metal Plug-In
}

\author{
Christian Koenen, Student Member, IEEE, Uwe Siart, Member, IEEE, Thomas F. Eibert, Senior Member, IEEE,
}

Garrard D. Conway, and Ulrich Stroth

\begin{abstract}
The design of a WR 10 waveguide coupling structure is presented. Electrically it consists of an E-plane (series) Tjunction and a quarter-wave section. The resulting coupling factor is nearly constant over the entire $W$-band. A milled split-block network and a wire-eroded metal plug-in, which is inserted into a groove in the split-block, implement the coupling structure. This modular design makes the coupling factor easily configurable and enables the realization of quarter-wave section heights in the range of tens of micrometers. The plug-in is fabricated by wire erosion, a machining technique based on electrical discharge (spark machining). This enables precise adjustment of the height and length of the quarter-wave section $( \pm 2 \mu \mathrm{m}$ contour accuracy) and provides polished-like surface quality $\left(R_{\mathrm{Z}}=\mathbf{2 . 1} \mu \mathrm{m}\right)$. Hints for the manufacturing of the metal plug-in are given, full-wave simulations as well as measurements of $W$-band prototype structures are evaluated and the possibility of a post-fabrication adjustment of the metal plug-ins is shown.
\end{abstract}

Index Terms-Millimeter-wave passive component, hollow waveguide components, modular waveguide structures

\section{INTRODUCTION}

I $\mathrm{N}$ order to realize the amplitude taper of a series-fed array antenna, a coupling structure is required to tap-off a defined amount of power from its trunk line. The coupling should be constant over the operational frequency range, as otherwise amplitude errors would occur which in general do not replicate the desired radiation characteristic. Coupling holes and slots are frequently used to set the amplitude distribution [1], [2]. However, at millimeter-wave frequencies these structures are difficult to realize with the required accuracy. Beyond that, they exhibit a relatively strong frequency dependence [3]. To tackle these problems, a coupling structure has been developed and published in [4]. Electrically it consists of a rectangular hollow waveguide E-plane (series) T-junction and a quarter-wave transformer section which controls the coupling factor. Almost frequency independent coupling values between $(-25.0 \pm 0.5) \mathrm{dB}$ and $(-10.0 \pm 0.6) \mathrm{dB}$ over the entire $W$-band $(75 \mathrm{GHz}$ to $110 \mathrm{GHz})$ were achieved. The coupling structure was designed with two pieces in order to achieve a

Manuscript received November 21, 2016; revised February 7, 2017

Funded by the Helmholtz Association of German Research Centers within the Helmholtz Virtual Institute on "Plasma Dynamical Processes and Turbulence Studies using Advanced Microwave Diagnostics". The authors are much obliged to Johannes Friesen and Daniel Grossmann.

C. Koenen, U. Siart, and T. F. Eibert are with the Chair of High-Frequency Engineering of the Technical University of Munich, 80290 München, Germany

G. D. Conway and U. Stroth are with the Max Planck Institute for Plasma Physics, 85748 Garching, Germany configurable coupling factor and in order to realize quarterwave section heights in the range of tens of micrometers. In [4], the WR 10 waveguide $(2.54 \mathrm{~mm} \times 1.27 \mathrm{~mm})$ was manufactured in split-block technology and the quarter-wave section was realized by a separate and exchangeable metal plug-in which was laser-cut from a metal sheet and inserted into the split-block. Thereby it was possible to adjust and tune the coupling factor by the height of the laser-cut quarterwave section and to modify the coupling value without the need to machine the whole milled split-block again. While laser-cutting is relatively inexpensive compared to milling of the split-block serial feed network, it has three inevitable drawbacks. The realized slots are undesirably tapered due to widening of the laser beam beyond the focal point. This induces deviations between the predicted and the actually achieved coupling factors. Furthermore, there is a minimum amount of material that gets dissipated. This limits the lowest value of the coupling factor which can be achieved by this manufacturing technology. The last issue is concerned with the length of the slot. In the laser-cut case it is fixed and given by the thickness of the metal sheet and the fitting groove in the split-block. This renders it impossible to optimize the slot lengths for different slot heights. However, it is sometimes necessary to implement very small coupling factors for the outermost elements of a series-fed array antenna and also to adjust the slot length according to the slot height to achieve the best performance.

This contribution puts forward an improved manufacturing technology for the coupling structure. It is again manufactured in two pieces: the milled WR 10 waveguide split-block network equipped with a groove and a separate metal plugin which is inserted into the groove and, thus, completes the coupling structure, see Fig. 1. The plug-in is now manufactured by wire erosion (a sort of electrical discharge / spark machining) to overcome the drawbacks of laser-cutting. In the following, the new design of the coupling structure with the wire-eroded metal plug-in is explained. Simulation results and measurements of a manufactured prototype with different coupling factors are presented and the possibility of postfabrication adjustment of the plug-ins is shown.

\section{DESIGN}

The considered rectangular hollow waveguide based coupling structure is illustrated in Fig. 1. An E-plane (series) Tjunction and a quarter-wave transformer section are the crucial 


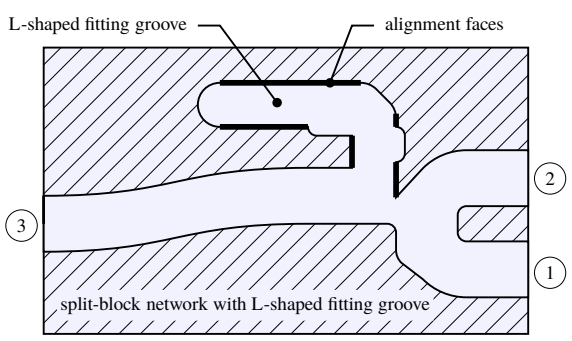

(a)

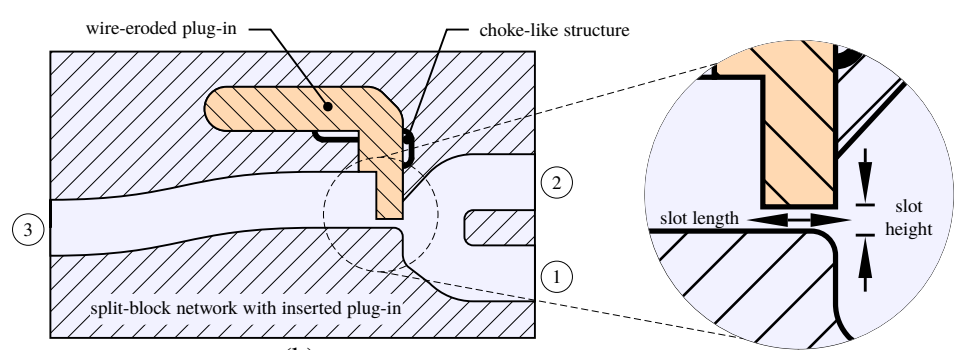

(b)

Figure 1. Drawing a) represents structure with an empty L-shaped groove and b) shows an inserted wire-eroded plug-in that models the coupling slot.

parts of the proposed structure. Their precision is of vital importance for the desired frequency independent coupling factor [4]. The T-junction is part of a hairpin waveguide bend, see Fig. 1 . The quarter-wave section is implemented by a metal plug-in which is fabricated from stainless steel and inserted into a milled groove in the split-block network, see Fig. 2. The waveguide from port 1 to port 2 in Fig. 1 is part of the trunk line of a series-fed array antenna and port 3 is the coupled port which feeds the radiating element. The plug-in shape is derived from a two-dimensional contour which is extruded in the third dimension. This is required for fabrication by wire erosion.

The L-shaped groove in the split-block guarantees precise positioning of the plug-in. Only the straight sections of the L-shaped groove are dedicated for alignment. The radii inside the fitting groove (thin lines) are dimensioned such that they do not contact those of the metal plug-in. Two choke-like structures placed a quarter-wavelength apart from the WR 10 waveguide (emphasized in Fig. 1) eliminate the impact of poor electrical contact between the milled split-block and the inserted wire-eroded plug-in. The nose-piece of the plugin reaches into the rectangular waveguide and shapes the quarter-wave section with a specific waveguide slot height and slot length (see Fig. 1b). With this design, it would also be possible to realize a second quarter wave transformer stage to further improve the coupling behavior. The wire erosion process at our workshop can achieve contour accuracy in the order of $\pm 2 \mu \mathrm{m}$. The smallest viable radius is $100 \mu \mathrm{m}$. It is essentially determined by the wire diameter. The surface roughness of the wire-eroded faces has been measured and it can be specified by $R_{\mathrm{Z}}=2.1 \mu \mathrm{m}$, which enumerates the average distance between the highest peak and the lowest valley in each sampling length. This surface texture is comparable to a moderately polished surface. It is significantly better than what was formerly obtained by laser cutting. Wire erosion starts and ends at a certain point on the contour, where an unavoidable residual nose remains. To avoid any influence of this nose on the coupling factor, this point is placed in one of the choke structures, as shown in Fig. 3.

Poor electrical contact between metal plug-in and splitblock will result in parasitic leakage and degraded coupling performance. Therefore, the plug-in height is a very critical dimension. Chokes are not feasible here as the plug-in contour may vary. In order to avoid such problems, our manufacturing process is as follows. First, a stainless-steel sheet is lapped to the desired thickness, which is $2.54 \mathrm{~mm}$ or some micrometers

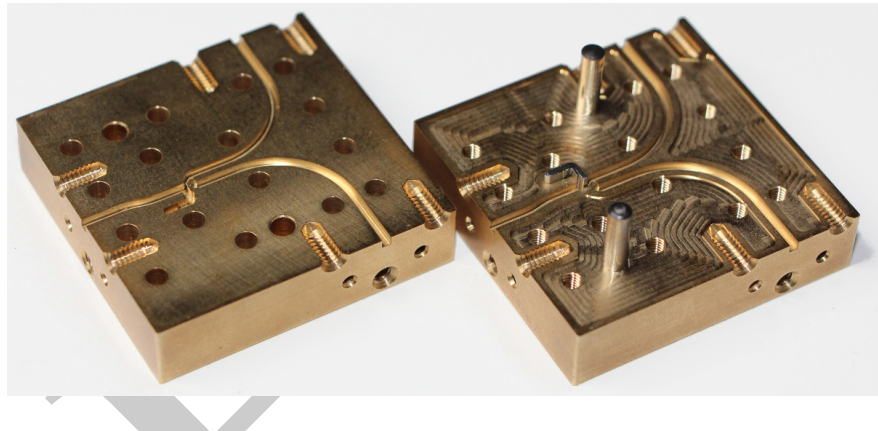

Figure 2. Image of the split-block network and inserted wire-eroded plug-in.

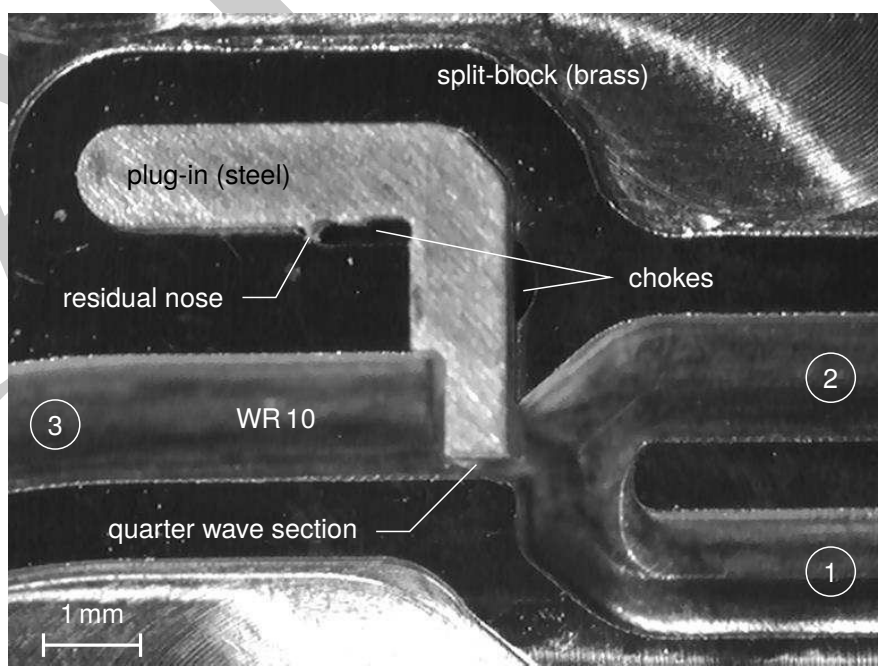

Figure 3. Microscope image of the 3-port coupling structure with the metal plug-in inserted. Port 1 is input, port 2 is through, and port 3 is coupled.

more for a $W$-band waveguide, and afterwards, the plug-in is wire-eroded from this sheet. An interference fit of the metal plug-in between the two split-blocks is preferred to a transition fit, as the plug-in gets slightly pressed into the milled groove and establishes good contact.

\section{Simulation and Measurement Results}

A photograph of the manufactured prototype is shown in Fig. 2. The split-block was milled from brass CW614N $(\mathrm{CuZn} 39 \mathrm{~Pb} 3)$ while the wire-eroded plug-ins were fabricated from stainless steel 1.4301 (X5CrNi18-10). For prototyping and testing, three different plug-ins and the coupling structure body were manufactured. They realize coupling factors within 
Table I

MANUFACTURED COUPLING SLOTS AND ACHIEVED COUPLING FACTORS (MEAN AND MAXIMUM DEVIATIONS).

\begin{tabular}{ccccc}
\hline $\begin{array}{c}\text { slot } \\
\text { height }\end{array}$ & $\begin{array}{c}\text { slot } \\
\text { length }\end{array}$ & $\begin{array}{c}\left|s_{31}\right|(\mathrm{dB}) \\
\text { simulated }\end{array}$ & $\begin{array}{c}\left|s_{31}\right|(\mathrm{dB}) \\
\text { measured } \\
\text { after fabrication }\end{array}$ & $\begin{array}{c}\left|s_{31}\right|(\mathrm{dB}) \\
\text { measured } \\
\text { after reworking }\end{array}$ \\
\hline $200 \mu \mathrm{m}$ & $635 \mu \mathrm{m}$ & $-14.5_{-0.2}^{+0.6}$ & $-14.8_{-0.3}^{+0.4}$ & $-14.6_{-0.3}^{+0.7}$ \\
$80 \mu \mathrm{m}$ & $763 \mu \mathrm{m}$ & $-21.8_{-0.2}^{+0.6}$ & $-22.5_{-0.3}^{+0.6}$ & $-21.7_{-0.3}^{+0.6}$ \\
$25 \mu \mathrm{m}$ & $865 \mu \mathrm{m}$ & $-31.5_{-0.2}^{+0.5}$ & $-35.5_{-1.3}^{+1.0}$ & $-30.9_{-0.9}^{+0.9}$ \\
\hline
\end{tabular}

the range from $-31 \mathrm{~dB}$ to $-14 \mathrm{~dB}$. Each slot length has been numerically optimized based on full-wave simulations using version 2016.4 of CST Microwave Studio ${ }^{\circledR}$. The optimization goal was maximum flatness over the operating frequency range from $75 \mathrm{GHz}$ to $105 \mathrm{GHz}$. The upper frequency limit is due to the targeted application in a Doppler reflectometry system. Manufactured slot heights and lengths with the associated mean coupling factors are listed in Table I. The coupling factors are compared for full-wave simulations, measurements of the fabricated pieces (out of the workshop, no modifications - just cleaning) and measurements with reworked metal plugins. Apart from the mean coupling values, the maximum deviations from the mean value over the frequency range from $75 \mathrm{GHz}$ to $105 \mathrm{GHz}$ are given. Measurements were carried out on an HP 8510C network analyzer with millimeter-wave extensions and a thru-reflect-line calibration (the measurement of a standard waveguide section after calibration showed an $s_{11} \leq-36 \mathrm{~dB}$ ). The mean coupling factor of the prototype network after fabrication is always below that of the simulated model. For smaller slot heights the difference gets larger. This difference is due to a smaller realized slot height where the error is below $10 \mu \mathrm{m}$ (modeled and verified with full-wave simulations). The transmission from port 1 to port 2 is around $-0.5 \mathrm{~dB}$. The main reason for this are the Ohmic losses in the $\sim 50 \mathrm{~mm}$ waveguide from the measurement flange to the coupling structure and vice versa, see Fig. 2. With reference planes $1 \mathrm{~mm}$ apart from the coupling structure, the $s_{21}$ is around $-0.2 \mathrm{~dB}$ for the $200 \mu \mathrm{m}$-slot and is basically driven by the $s_{31}$. With the new design it is possible to accurately realize very low coupling factors just by fabrication and cleaning. The error of the mean coupling is $-0.3 \mathrm{~dB}$ in case of the $-14.5 \mathrm{~dB}$ coupling factor. The $-21.8 \mathrm{~dB}$-slot deviates by $-0.7 \mathrm{~dB}$. In both cases, the coupling factor variations have no mentionable difference. In contrast, the measurements of the $25 \mu \mathrm{m}$-slot do not agree well with the simulation. Here, manufacturing tolerances (approx. $40 \%$ of the slot height) have a very strong impact. The fifth column in Table I represents the measurement of reworked metal plug-ins. The goal was to get closer to the simulated coupling factor, what required to enlarge the realized slot height. For this, the nose piece of the plugins has been tuned with abrasive paper (400 grit). After two iterations of careful sanding, the two strongest coupling factors deviate by $0.1 \mathrm{~dB}$ from the simulated values. Again, tuning the smallest slot is more challenging because modifications have a stronger impact on the coupling factor. Nevertheless, there

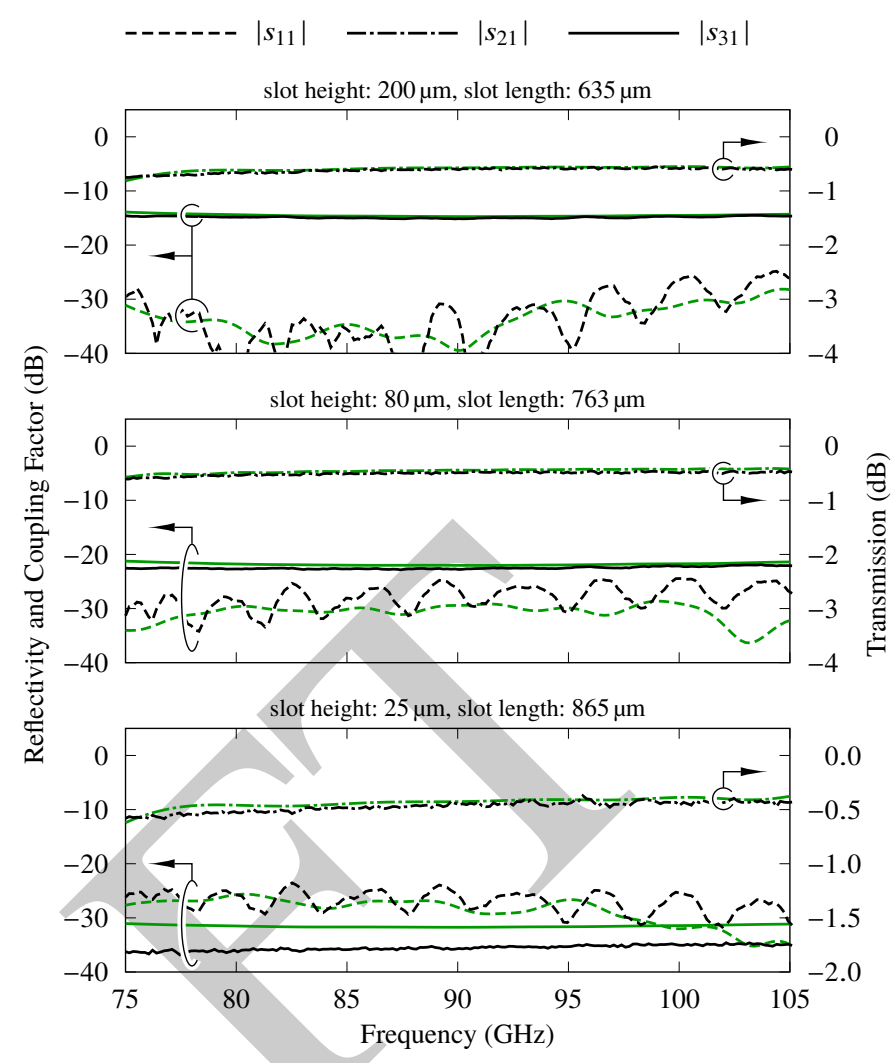

Figure 4. Simulated (green) and measured (black) S-parameters for slots defined in Table I. These plots represent the measurement directly after fabrication $\left(4^{\text {th }}\right.$ column in Table I).

is no need to rework the milled split-block in order to mitigate manufacturing tolerances and adjust the coupling factor to the desired value.

\section{CONCLUSION}

The design of a broadband hollow waveguide coupling structure was presented. It is based on an E-plane T-junction and a quarter-wave section which determines the coupling factor. This coupling factor can be accurately configured by a purpose-built metal plug-in. The plug-in was manufactured by wire erosion to achieve precise quarter-wave section heights and a good surface. Even very low coupling values on the order of $-30 \mathrm{~dB}$ were achieved. Deviations between simulated and measured coupling values are small. They are mostly rooted in height discrepancy of the quarter-wave section at the scale of manufacturing tolerances. These errors could be eliminated by carefully reworking and fine tuning of the metal plug-ins.

\section{REFERENCES}

[1] W. H. Watson, "Resonant slots," Journal of the Institution of Electrical Engineers - Part IIIA: Radiolocation, vol. 93, no. 4, pp. 747-777, 1946.

[2] A. F. Stevenson, "Theory of slots in rectangular wave-guides," Journal of Applied Physics, vol. 19, no. 1, p. 24, 1948.

[3] P. Rohmann, S. Wolf, W. Kasparek, B. Plaum, and J. Hesselbarth, "A 32element frequency-steered array antenna for reflectometry in W-band," in IEEE Int. Symp. Phased Array Systems and Technology, Waltham, MA, USA, Oct 2013, pp. 559-563.

[4] C. Koenen, U. Siart, T. F. Eibert, G. D. Conway, and U. Stroth, "A configurable coupling structure for broadband millimeter-wave split-block networks," IEEE Trans. Microw. Theory Techn., vol. 63, no. 12, pp. 39543961, December 2015. 